\title{
Gaussian Wave Functions for Polyatomic Molecules: Integral Formulas
}

\author{
Morris Krauss
}

(November 22, 1963)

\section{Introduction}

Gaussian wave functions have found increasingly wide application in recent years for molecular energy calculations [1]. ${ }^{1} \quad$ This is due to the relative ease of computation of the three- and fourcenter coulomb integrals; a circumstance that was first noted by Boys [2] and McWeeny [3]. Boys determined explicit formulas for all the relevant molecular integrals involving $1 s$ Gaussian functions centered at arbitrary points in space. He also observed that all other integrals could be obtained by differentiation of the resulting formulas. Harris noted that this procedure would be very tedious and he extended the explicit algebraic representation of the integrals to cases where the principal quantum number of the Gaussian function equals the azimuthal quantum number [4]. However, when the principal quantum number exceeded the azimuthal one the integrals were left as functions of differential operators. In this paper explicit formulas will be obtained for all cases by a different procedure than that used by Harris.

Both the one- and two-electron coulomb integrals will be considered. The nuclear attraction integral is

$$
\left(A n l m\left|r_{C}^{-1}\right| B n^{\prime} l^{\prime} m^{\prime}\right)=\int d \tau \psi_{n l m}^{A *}(\vec{r}) \psi_{n^{\prime} l^{\prime} m^{\prime}}^{B}(\vec{r}) r_{C}^{-1}
$$

and the electron repulsion integral is

$$
\begin{gathered}
\left(A n l m B n^{\prime} l^{\prime} m^{\prime} \mid C n^{\prime \prime} l^{\prime \prime} m^{\prime \prime} D n^{\prime \prime \prime} l^{\prime \prime \prime} m^{\prime \prime \prime}\right) \\
=\int d \tau_{1} d \tau_{2} \psi_{n l m}^{A *}\left(\overrightarrow{r_{1}}\right) \psi_{n^{\prime} l^{\prime} m^{\prime}}^{B}\left(\overrightarrow{r_{1}}\right) \psi_{n^{\prime \prime} l^{\prime \prime \prime} m^{\prime \prime}}^{C *}\left(\overrightarrow{r_{2}}\right) \psi_{n^{\prime \prime \prime} l^{\prime \prime \prime} m^{\prime \prime \prime}}^{D}\left(\overrightarrow{r_{2}}\right) r_{12}{ }^{-1},
\end{gathered}
$$

where

$$
r_{12}=\left|\overrightarrow{r_{1}}-\vec{r}_{2}\right| \text { and } r_{C}=|\vec{r}-\vec{C}| \text {. }
$$

The usual expansion of the function $r_{12}{ }^{-1}$ in spherical [5] or cylindrical coordinates [6] is rejected in favor of the relation [7]

$$
r^{-1}=\frac{1}{2 \pi^{2}} \int d \vec{k} k^{-2} \exp [i \vec{k} \cdot \vec{r}]
$$

This representation is favorable for the evaluation of coulomb integrals with basis functions of the following form

$$
\psi_{n l m}^{A}(\vec{r})=r_{A}^{2 n+l} P_{l}^{|m|}\left(\cos \theta_{A}\right) e^{i m \phi_{A}^{-a r}{ }^{2}}
$$

where $\left(r_{A}, \theta_{A}, \phi_{A}\right)$ are spherical polar coordinates with origin at the point $A$ and $P_{l}|m|(x)$ is the associated Legendre spherical harmonic [8]. It will be seen that the use of eq (3) essentially reduces the task of evaluating the coulomb integral to the determination of the three-dimensional Fourier transform of $\psi_{n l m}^{A}$.

${ }^{1}$ Figures in brackets indicate the literature references at the end of this paper. 
It is convenient first to reduce the dependence of the coulomb integral on molecular geometry by referring the coordinate axes at the atom centers to a new system with a common orientation. This was done by Harris. Such a transformation will be discussed briefly in the second section of this paper. By these means we isolate the basic integrals, whose evaluation is the main concern of this paper.

The Fourier transform of $\psi_{n l m}^{A}$ is readily found in section 3. The remaining integrations over the $k$ variables are then completed for the nuclear attraction integral in section 3 and for the coulomb repulsion integral in section 4. The use of the Gaussian functions is briefly discussed in section 5 .

\section{Transformation of the Integrals}

It was shown by Boys that it is convenient to expand the product $\psi_{n l m}^{A *} \psi_{n^{\prime} l^{\prime} m^{\prime}}^{B}$ in eqs (1) and (2) at a point $P$ on the directed line between $A$ and $B$. Similarly, the product $\psi_{n^{\prime \prime} l^{\prime \prime \prime} m^{\prime \prime}}^{*} \psi_{n^{\prime \prime \prime} l^{\prime \prime \prime} m^{\prime \prime \prime}}^{D}$ is expanded about $Q$. The polar axes of the coordinate systems at points $P$ and $Q$ are initially along $B-A$ and $D-C$, respectively. The coordinate system at $P$ is obtained from the one at $A$ by rotation through the Euler angles $(\alpha, \beta, \gamma)$, collectively characterized as $(P A)$, and translation without rotation a distance $|P-A|=b R_{1} /(a+b)$.

The basis functions in eq (2) are then transformed by means of the following expressions.

The rotation of the spherical harmonic through the Euler angles $\alpha, \beta, \gamma$ is given by [9].

$$
P_{l}^{|m|}(\cos \theta) l^{i m \phi}=\sum_{m^{\prime}=-l}^{l} D_{l}^{m m^{\prime}}(\alpha, \beta, \gamma) P_{e^{\left|m^{\prime}\right|}}\left(\cos \theta^{\prime}\right) e^{i m^{\prime} \phi^{\prime}}
$$

When the spherical harmonic is translated without rotation a distance $t$ along the polar axis, it is transformed [10]

$$
r^{l} P_{l}^{|m|}(\cos \theta)=\sum_{j=|m|}^{l}\left(\begin{array}{c}
l+|m| \\
j+|m|
\end{array}\right) t^{l-j} r^{\prime j} P_{j}^{|m|}\left(\cos \theta^{\prime}\right) .
$$

Also, the trinomial expansion will yield

$$
\begin{gathered}
r_{A}{ }^{2 n}=\left(r_{P}^{2}+\frac{2 b}{a+b} R_{1} r_{P} \cos \theta_{P}+\frac{b^{2}}{(a+b)^{2}} R_{1}{ }^{2}\right)^{n} \\
=\sum_{\substack{\left(\sum_{j}=n\right) \\
i}} \frac{n !}{n_{1} ! n_{2} ! n_{3} !}\left(\frac{2 b}{a+b} R_{1}\right)^{n}{ }^{2}\left(\frac{b}{a+b} R_{1}\right)^{2 n}{ }^{2 n} r_{P}{ }^{2 n_{1}}\left(r_{P} \cos \theta_{P}\right)^{n_{2}} .
\end{gathered}
$$

The use of eqs (5), (6), and (7) permit the expansion of the product $\psi_{n l m}^{A *} \psi_{n^{\prime} l^{\prime} m^{\prime}}^{B}$ in terms of the coordinate system centered at $P$. Included in this expansion is a product of two Legendre polynomials which may be expanded [11]

$$
P_{j}^{|m|}(x) P_{j^{\prime}}^{|m|}(x)=\sum_{J} C_{J j j^{\prime}}^{m m^{\prime}} P_{J}^{m^{\prime}-m}(x) .
$$

We also note that by multiple use of the recurrence formula [12] for the Legendre polynomials we can obtain

$$
x^{j} P_{k}^{|m|}(x)=\sum_{n=-j}^{j} a_{n} P_{k+n}^{|m|}(x)
$$

where $a_{n}=0$ if $n=-j+2 r+1$ for integer $r$. The $a_{n}$ can easily be determined for any particular case. A similar series of transformations is applied in the coulomb repulsion integral to transform the product $\psi_{n^{\prime \prime} l^{\prime \prime} m^{\prime \prime}}^{C *} \psi_{n^{\prime \prime \prime} l^{\prime \prime \prime} m^{\prime \prime},}^{D} ;$ the coordinate system is centered at $Q$.

The last step in transforming the integrands for both the nuclear attraction and coulomb repulsion integrals is to rotate the coordinate axis of $P$ in the first instance to have its polar axis directed along $C-P$ and for the coulomb repulsion integral the axes at $P$ and $Q$ are rotated to have a common orientation with the polar axis $Q-P$. In both these cases the Euler angles for the rotation at $P$ will be denoted by $\left(P^{\prime} P\right)$. These results and the definitions in the appendix allow us to factor out the dependence on molecular geometry from eqs (1) and (2). 


\section{Nuclear Attraction Integral}

The nuclear attraction integral can be written

$$
\begin{aligned}
& \left(A n l m\left|r_{C}{ }^{-1}\right| B n^{\prime} l^{\prime} m^{\prime}\right)=e^{-x_{1}} \sum_{\sigma=-l}^{l} \sum_{\sigma^{\prime}=-l^{\prime}}^{l^{\prime}} D_{l}^{m \sigma *}(P A) D_{l^{\prime}} m^{\prime} \sigma^{\prime}(P B) \\
& \sum_{j=|\sigma|}^{l} \sum_{j^{\prime}=\left|\sigma^{\prime}\right|}^{l^{\prime}} \sum_{\substack{\sum \\
i}} \sum_{i=n)} \sum_{\substack{\left.\sum n^{\prime}=n^{\prime}\right) \\
i}}\left(\begin{array}{l}
l+|\sigma| \\
j+|\sigma|
\end{array}\right)\left(\begin{array}{l}
l^{\prime}+\left|\sigma^{\prime}\right| \\
j^{\prime}+\left|\sigma^{\prime}\right|
\end{array}\right)\left(\frac{b}{(a+b)} R_{1}\right)^{l-j}\left(-\frac{a}{(a+b)} R_{1}\right)^{l^{\prime}-j^{\prime}} \\
& \frac{n ! n^{\prime} !}{n_{1} ! n_{2} ! n_{3} ! n_{1}^{\prime} ! n_{2}^{\prime} ! n_{3}^{\prime} !}\left(\frac{2 b}{a+b} R_{1}\right)^{n_{2}}\left(-\frac{2 a}{a+b} R_{1}\right)^{n_{2}^{\prime}}\left(\frac{b}{a+b} R_{1}\right)^{2 n_{3}}\left(\frac{a}{a+b} R_{1}\right)^{2 n_{3}^{\prime}} \\
& \sum_{J} C_{J j j^{\prime}} \sigma \sigma^{\prime} \sum_{k=-\left(n_{2}+n_{2}^{\prime}\right)}^{n_{2}+n_{2}^{\prime}} a_{k} \sum_{M=-(J+k)}^{J+k} D_{J+k}\left(\sigma^{\prime}-\sigma\right), M\left(P^{\prime} P\right) \\
& \int d \tau r_{P}^{\prime j+j^{\prime}+2\left(n_{1}+n_{1}\right)+n_{2}+n_{2}^{\prime} P_{J+k}|M|}\left(\cos \phi_{P}^{\prime}\right) e^{i M \phi_{P}^{\prime}-(a+b) r_{P}^{\prime 2}} r_{C}^{-1} .
\end{aligned}
$$

It is readily seen that we must evaluate the integral

$$
I_{N L}^{M}=\int d \tau r_{P}^{2 N+L} P_{L}^{|M|}\left(\cos \phi_{P}\right) e^{i M \phi_{P}-U^{2} r_{P}^{2} r_{C}{ }^{-1^{\prime}}}
$$

The primes have been dropped in eq (4) but the coordinate system has its polar axis along $C-P$ and its origin at $P$. Although $M=0$ in this coordinate system from symmetry considerations, we shall nonetheless retain the factor $P_{L^{|M|}}(\cos \phi) e^{i M \phi}$ since the more general expression is required for the electron repulsion integral. Substituting (3) into eq (11) we obtain

$$
\begin{aligned}
I_{N L}^{M}= & \frac{1}{2 \Pi^{2}} \int \overrightarrow{d k} k^{-2} e^{\overrightarrow{i k} \cdot C-P} . \\
& \int d \tau_{P} r_{P}{ }^{2 N+L} P_{L}{ }^{M}\left(\cos \phi_{P}\right) e^{-U^{2} r_{P}^{2}+i M \phi+i \vec{k} \cdot \vec{r}_{P}} .
\end{aligned}
$$

Without loss of generality $\phi_{k}$ can be set equal to zero, so that

$$
\exp \left[\overrightarrow{i k} \cdot \vec{r}_{P}\right]=\exp \left[i k r_{P}\left(\cos \theta_{P} \cos \psi-i \sin \theta_{P} \sin \psi \cos \phi_{P}\right)\right]
$$

where $\psi$ is the angle between the $C P$ axis and $\vec{k}$. Consider

$J=\int_{0}^{\infty} d r_{P} r_{P}^{2 N+L+2} \int_{0}^{\pi} \int_{0}^{2 \pi} d \theta_{P} d \phi_{P} \sin \theta_{P} P_{L^{M}}\left(\cos \theta_{P}\right)$

$$
\exp \left[i r_{P}\left(\cos \theta_{P} \cos \psi+\sin \theta_{P} \sin \psi \cos \phi_{P}\right)+i M \phi_{P}-U^{2} r_{P}{ }^{2}\right]
$$

and integrate over the angular coordinates to obtain

$$
J=(2 \pi)^{3 / 2} \frac{i^{L}}{k^{1 / 2}} P_{L}^{M}(\cos \psi) \int_{0}^{\infty} d r_{P} r_{P}^{2 N+L+3 / 2} J_{L+1 / 2}\left(k r_{P}\right) e_{P}^{-U^{2} r^{2}}
$$

Utilizing the Weber and Sonine formula [13]

$$
\int_{0}^{\infty} d t J_{\nu}(a t) e^{-p^{2} t^{2}} t^{\mu-1}=\frac{\left(\frac{a}{2 p}\right)^{\nu} \Gamma\left(\frac{\mu+\nu}{2}\right)}{2 p^{\nu} \Gamma(\nu+1)}{ }_{1} F_{1}\left(\frac{\mu+\nu}{2} ; \nu+1 ;-\frac{a^{2}}{4 p^{2}}\right)
$$


we obtain finally

$$
J=\frac{\pi^{3 / 2}}{2^{L}} i^{L} \frac{\Gamma(N+L+3 / 2)}{\Gamma(L+3 / 2) U^{2 N+2 L+3}} P_{L^{M}}(\cos \psi) k^{L}{ }_{1} F_{1}\left(\mathrm{~N}+L+3 / 2 ; L+3 / 2 ;-\frac{k^{2}}{4 U^{2}}\right) .
$$

Substituting $J$ into eq (12) and setting $M=O$, we obtain

$$
\begin{gathered}
I_{N L}=\frac{i^{L}}{2^{L+1} \pi^{1 / 2}} \frac{\Gamma(N+L+3 / 2)}{\Gamma(L+3 / 2) U^{2 N+2 L+3}} \\
\int d \vec{k} k^{L-2} P_{L}(\cos \psi)_{1} F_{1}\left(N+L+3 / 2 ; L+3 / 2 ;-\frac{k^{2}}{4 U^{2}}\right) \exp [\overrightarrow{i k} \cdot \overrightarrow{C P}] .
\end{gathered}
$$

Integration over the angles yields

$$
I_{N L}=\frac{C}{|C P|^{1 / 2}} \int_{0}^{\infty} d k k^{L-1 / 2} J_{L+1 / 2}(k|C P|)_{1} F_{1}\left(N+L+3 / 2 ; L+3 / 2 ;-\frac{k^{2}}{4 U^{2}}\right)
$$

with

$$
C=2^{-L+1 / 2} \pi \frac{\Gamma(N+L+3 / 2)}{\Gamma(L+3 / 2) U^{2 N+2 L+3}} .
$$

This integral, considered as a Hankel transform [14], is given as

$$
I_{N L}=\frac{\pi}{|C P|^{L+1} U^{2 N+2 L+3}} G_{23}^{21}\left(U^{2}|C P|^{2} \mid \begin{array}{c}
1, L+3 / 2 \\
L+1 / 2, N+L+3 / 2,0
\end{array}\right)
$$

where the $G$ function is a polynomial in generalized hypergeometric functions [15].

An alternative formula may be obtained by relating the confluent hypergeometric function to the Laguerre polynomial [16],

$$
{ }_{1} F_{1}\left(N+L+3 / 2 ; L+3 / 2 ;-\frac{k^{2}}{4 U^{2}}\right)=\frac{N !}{(L+3 / 2)_{N}} e^{-k^{2} / 4 U^{2}} L_{N}^{L+1 / 2}\left(\frac{k^{2}}{4 U^{2}}\right)
$$

and $(a)_{L}=(a)(a+1) \ldots(a+L-1)$.

The Laguerre function is given as the power series [16]

$$
L_{N}^{L+1 / 2}\left(\frac{k^{2}}{4 U^{2}}\right)=\sum_{S=0}^{N}(-)^{S}\left(\begin{array}{c}
N+L+1 / 2 \\
N-S
\end{array}\right) \frac{k^{2 S}}{(2 U)^{2 S} S !}
$$

and therefore

$$
\begin{aligned}
I_{N L}= & \frac{C}{|C P|^{1 / 2}} \frac{N !}{(L+3 / 2)_{N}} \sum_{S=0}^{N}(-)^{S}\left(\begin{array}{c}
N+L+1 / 2 \\
N-S
\end{array}\right) \frac{1}{(2 U)^{2 S} S !} \\
& \int_{0}^{\infty} d k k^{L+2 S-1 / 2} J_{L+1 / 2}(k|C P|) e^{-k^{2} / 4 U^{2}} \\
= & \pi \frac{N !}{\Gamma(L+3 / 2)} \frac{|C P|^{L}}{U^{2(N+1)}} \sum_{S=0}^{N} \frac{(-)^{S}}{S !}\left(\begin{array}{c}
N+L+1 / 2 \\
N-S
\end{array}\right)
\end{aligned}
$$

$\Gamma(L+S+1 / 2) \exp \left(-U^{2}|C P|^{2}\right)_{1} F_{1}\left(-S+1 ; L+3 / 2 ; U^{2}|C P|^{2}\right)$. 


\section{Coulomb Repulsion Integral}

The coulomb integral, in the most general case, will reduce to $\left[A n l m B n^{\prime} l^{\prime} m^{\prime} \mid C n^{\prime \prime} l^{\prime \prime} m^{\prime \prime} D n^{\prime \prime \prime} l^{\prime \prime \prime} m^{\prime \prime \prime}\right]=e^{-X_{1}-X_{2}}$

$$
\begin{aligned}
& \sum_{\sigma=-l}^{l} \sum_{\sigma^{\prime}=-l^{\prime}}^{l^{\prime}} \sum_{\sigma^{\prime \prime}=-l^{\prime \prime}}^{l^{\prime \prime}} \sum_{\sigma^{\prime \prime \prime}=-l^{\prime \prime \prime}}^{l^{\prime \prime \prime}} D_{l}^{m \sigma *}(P A) D_{l^{\prime}}^{m^{\prime} \sigma^{\prime}}(P B) D_{l^{\prime \prime}}^{m^{\prime \prime} \sigma^{\prime \prime} *}(Q C) D_{l^{\prime \prime \prime}}^{m^{\prime \prime \prime} \sigma^{\prime \prime \prime}}(Q D) \\
& \sum_{j=|\sigma|}^{l} \sum_{j^{\prime}=\left|\sigma^{\prime}\right|}^{l^{\prime}} \sum_{j^{\prime \prime}=\left|\sigma^{\prime \prime}\right| j^{\prime \prime \prime}=\left|\sigma^{\prime \prime \prime}\right|}^{l^{\prime \prime}} \sum_{a+b}^{l^{\prime \prime \prime}}\left(\frac{b R_{1}}{a+b}\right)^{l-j}\left(\frac{-a R_{1}}{a+b}\right)^{l^{\prime}-j^{\prime}}\left(\frac{d R_{2}}{C+d}\right)^{l^{\prime \prime}-j^{\prime \prime}}\left(\frac{-C R_{2}}{C+d}\right)^{l^{\prime \prime \prime}-j^{\prime \prime \prime}} \\
& \left(\begin{array}{l}
l+|\sigma| \\
j+|\sigma|
\end{array}\right)\left(\begin{array}{l}
l^{\prime}+\left|\sigma^{\prime}\right| \\
j^{\prime}+\left|\sigma^{\prime}\right|
\end{array}\right)\left(\begin{array}{l}
l^{\prime \prime}+\left|\sigma^{\prime \prime}\right| \\
j^{\prime \prime}+\left|\sigma^{\prime \prime}\right|
\end{array}\right)\left(\begin{array}{l}
l^{\prime \prime \prime}+\left|\sigma^{\prime \prime \prime}\right| \\
j^{\prime \prime \prime}+\left|\sigma^{\prime \prime \prime}\right|
\end{array}\right) \sum_{\left(\sum n_{i}=n\right)} \sum_{\left(\sum n_{i}=n^{\prime}\right)} \sum_{\left(\sum n_{i}^{\prime \prime}=n^{\prime \prime}\right)} \sum_{\left(\sum n_{i}^{\prime \prime \prime}=n^{\prime \prime \prime}\right)} \\
& \frac{n ! n^{\prime} ! n^{\prime \prime} ! n^{\prime \prime \prime} !}{n_{1} ! n_{2} ! n_{3} ! n_{1}^{\prime} ! n_{2}^{\prime} ! n_{3}^{\prime} ! n^{\prime \prime}{ }_{1} ! n_{2}^{\prime \prime} ! n^{\prime \prime}{ }_{3} ! n^{\prime \prime}{ }_{1}^{\prime} ! n^{\prime \prime \prime}{ }_{2} ! n^{\prime \prime \prime}{ }_{3} !}\left(\frac{2 b R_{1}}{a+b}\right)^{n_{2}}\left(-\frac{2 a R_{1}}{a+b}\right)^{n_{2}^{\prime}} \\
& \left(\frac{2 d R_{2}}{c+d}\right)^{n_{2}^{\prime \prime}}\left(-\frac{2 c R_{2}}{c+d}\right)^{n_{2}^{\prime \prime \prime}}\left(\frac{b R_{1}}{a+b}\right)^{2 n_{3}}\left(\frac{a R_{1}}{a+b}\right)^{2 n_{3}^{\prime}}\left(\frac{d R_{2}}{c+d}\right)^{2 n_{3}^{\prime \prime}}\left(\frac{c R_{2}}{c+d}\right)^{2 n_{3}^{\prime \prime \prime}} \\
& \sum_{J} \sum_{J^{\prime}} C_{J j j^{\prime}}^{\sigma \sigma} C_{J^{\prime} j^{\prime} j^{\prime \prime \prime \prime}}^{\sigma^{\prime \prime \prime} \sigma^{\prime \prime \prime}} \sum_{k=-\left(n_{2}+n_{2}\right)}^{n_{2}+n_{2}^{\prime}} \sum_{k^{\prime}=-\left(n_{2}^{\prime \prime}+n_{2}^{\prime \prime \prime}\right)}^{n_{2}^{\prime \prime}+n_{2}^{\prime \prime \prime}} a_{p} a_{p^{\prime}} \sum_{M=-(J+k)-}^{(J+k)-} D_{J+k}^{\left(\sigma^{\prime}-\sigma\right),-M}\left(P^{\prime} P\right) D_{J^{\prime}+k^{\prime}}^{\left(\sigma^{\prime \prime \prime}-\sigma^{\prime \prime}\right), M}\left(Q^{\prime} Q\right)
\end{aligned}
$$

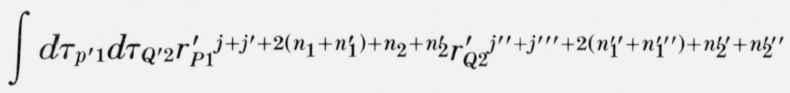

$$
\begin{aligned}
& P_{J+k}^{M}\left(\cos \theta_{P 1}^{\prime}\right) P_{J^{\prime}+k^{\prime}}^{M}\left(\cos \theta_{Q 2}^{\prime}\right) e^{i M\left(\phi_{Q 2}^{\prime}-\phi_{P 1}^{\prime}\right)-U^{2} r_{P 1}^{\prime 2}-V^{2} r_{Q 2}^{\prime 2}} \frac{1}{r_{12}}
\end{aligned}
$$

where $(J+k)_{-}$is the smallest of $(J+k)$ and $\left(J^{\prime}+k^{\prime}\right)$. Evidently we need to evaluate the following integral

$$
\begin{aligned}
I^{M}{ }_{N L N^{\prime} L^{\prime}}= & \int d \tau_{P 1} d \tau_{Q 2} r_{P 1}{ }^{-2 N+L} P_{L}{ }^{|M|}\left(\cos \theta_{P 1}\right) r_{Q 2}{ }^{2 N^{\prime}+L^{\prime}} P_{L^{\prime}}{ }^{|M|}\left(\cos \theta_{Q 2}\right) \\
& e^{i M\left(\phi_{Q 2}-\phi_{P 1}\right)-U^{2} r_{P 1}^{2}-V^{2} r_{Q 2}^{2}}
\end{aligned}
$$

where the polar axis is along $Q-P$. Again using the transformation of eq (1) we can separate the two-electron integral into a product of integrals each over only the coordinates of one electron. These integrals are, of course, very similar to the nuclear attraction integral that has just been evaluated. Therefore, we can immediately write down the integral over $k$.

$$
\begin{aligned}
I^{M}{ }_{N L N^{\prime} L^{\prime}}= & C \int d \vec{k} k^{L+L^{\prime}-2} P_{L}{ }^{M}(\cos \psi) P_{L^{\prime}}{ }^{M}(\cos \psi)^{e i \vec{k}(P-Q)} \\
& { }_{1} F_{1}\left(N+L+3 / 2 ; L+3 / 2 ;-k^{2} / 4 U^{2}\right)_{1} F_{1}\left(N^{\prime}+L^{\prime}+3 / 2 ; L^{\prime}+3 / 2 ;-k^{2} / 4 V^{2}\right)
\end{aligned}
$$

with

$$
C=(-)^{L^{\prime}} 2^{-L-L^{\prime}-1} \pi i^{L+L^{\prime}} \frac{\Gamma(N+L+3 / 2) \Gamma\left(N^{\prime}+L^{\prime}+3 / 2\right)}{U^{2 N+2 L+3} V^{2 N^{\prime}+2 L^{\prime}+3} \Gamma(L+3 / 2) \Gamma\left(L^{\prime}+3 / 2\right)}
$$


The angular integrations are readily performed using eq (8) and the relation [17]

$$
e^{i k r \cos \psi}=\sum_{l=0}(2 l+1) i{ }^{l} j_{l}(k r) P_{l}(\cos \psi)
$$

Equation (18) reduces to

$$
\begin{gathered}
I^{M}{ }_{N L N^{\prime} L^{\prime}}=4 \pi C \sum_{J} i^{J} C^{M M}{ }_{J L L^{\prime}} \int_{0}^{\infty} d k k^{L+L^{\prime}} j_{J}(k|P Q|) \\
{ }_{1} F_{1}\left(N+L+3 / 2 ; L+3 / 2 ;-k^{2} / 4 U^{2}\right)_{1} F_{1}\left(N^{\prime}+L^{\prime}+3 / 2 ; L^{\prime}+3 / 2 ;-k^{2} / 4 V^{2}\right) \\
=4 \pi C \sum_{J} i^{J} C^{M M}{ }_{J L L^{\prime}} I(J) .
\end{gathered}
$$

The confluent hypergeometric functions can again be given in terms of the associated Laguerre functions. We then find

$$
\begin{aligned}
I(J)=\frac{N ! N^{\prime} !}{(L+3 / 2)_{N}\left(L^{\prime}+3 / 2\right)_{N^{\prime}}} \sum_{S=0}^{N} \sum_{S^{\prime}=0}^{N^{\prime}}(-)^{S+S^{\prime}}\left(\begin{array}{c}
N+L+\frac{1}{2} \\
N-S
\end{array}\right)\left(\begin{array}{c}
N^{\prime}+L^{\prime}+\frac{1}{2} \\
N^{\prime}-S^{\prime}
\end{array}\right) \\
\frac{2^{-2\left(S+S^{\prime}\right)}}{S ! S^{\prime} !} U^{-2 S} V^{-2 S^{\prime}} \int_{0}^{\infty} d k k^{L+L^{\prime}+2\left(S+S^{\prime}\right)} j_{J}(k|P Q|)_{e}-\frac{k^{2}}{4}\left(\frac{U^{2}+V^{2}}{U^{2} V^{2}}\right) .
\end{aligned}
$$

For $\gamma^{2}=\frac{U^{2}+V^{2}}{U^{2} V^{2}}$, we again utilize the Weber and Sonine formula to obtain

$$
\begin{aligned}
& I(J)=\pi^{1 / 2} \frac{N ! N^{\prime} !}{(L+3 / 2)_{N}\left(L^{\prime}+3 / 2\right)_{N^{\prime}}} \frac{2^{L+L^{\prime}-1|P Q|^{J}}}{\Gamma(J+3 / 2)} \\
& \sum_{S=0}^{N} \sum_{S^{\prime}=0}^{N^{\prime}}(-)^{S+S^{\prime}} \frac{\Gamma\left(\frac{L+L^{\prime}+2\left(S+S^{\prime}\right)+J+1}{2}\right)}{\gamma^{L+L^{\prime}+J+2\left(S+S^{\prime}\right)+1}\left(\begin{array}{c}
N+L+1 / 2 \\
N-S
\end{array}\right)\left(\begin{array}{c}
N^{\prime}+L^{\prime}+1 / 2 \\
N^{\prime}-S^{\prime}
\end{array}\right)} \\
& \frac{U^{-2 S V^{-2 S^{\prime}}}}{S ! S^{\prime} !}{ }_{1} F_{1}\left(\frac{L+L^{\prime}+J+2\left(S+S^{\prime}\right)+1}{2} ; J+3 / 2 ;-\frac{|P Q|^{2}}{\gamma}\right) .
\end{aligned}
$$

The substitution of (20) into eq (19) completes the derivation of an explicit algebraic formula for the coulomb repulsion integral.

\section{Discussion}

The connection with the usual expression of these integrals in terms of error functions and derivatives of error functions may be seen from the representation of the error function by the confluent hypergeometric function [18]

$$
\frac{2}{\sqrt{\pi}} \int_{0}^{x} e^{-t^{2}} d t=\frac{2 x}{\sqrt{\pi}}{ }_{1} F_{1}\left(\frac{1}{2} ; \frac{3}{2} ;-x^{2}\right) .
$$

There is little reason, however, to replace the confluent hypergeometric functions with the more familiar error functions. For computational purposes the confluent hypergeometric functions are quite adequate. 
These formulas make it possible to extend the use of Gaussian basis functions to higher principal and azimuthal quantum numbers which is required if the Hartree-Fock limit is to be approached for most molecules. The integral computation time will not materially increase as the quantum number is increased; the major difficulty is the inherent inaccuracy of the Gaussians (i.e., the wrong cusp values and asymptotic behavior) and a large number of basis functions is required to overcome this deficiency. For example, for a simple system like $\mathrm{H}_{2}$ about twice as many Gaussian basis functions as Slater functions are required to achieve comparable results [19]. Therefore, the main problem remains the handling of the very large blocks of numbers that are generated in the Hartree-Fock procedure.

\section{Appendix I. Definition of Terms}

$$
\begin{array}{lr}
R_{1}=|A-B| & P=\frac{a A+b B}{a+b} \\
R_{2}=|C-D| & Q=\frac{c C+d D}{c+d} \\
X_{1}=\frac{a b R_{1}^{2}}{a+b} & U^{2}=a+b \\
X_{2}=\frac{c d R_{2}^{2}}{c+d} & V^{2}=c+d
\end{array}
$$

\section{References}

[1] R. K. Nesbet, J. Chem. Phys. 32, 1114 (1960); M. Krauss, J. Chem. Phys. 38, 564 (1963); C. M. Reeves and M. C. Harrison, J. Chem. Phys. 39, 11 (1963).

[2] S. F. Boys, Proc. Roy. Soc. (London) A200, 542 (1950).

[3] R. McWeeny, Nature 166, 21 (1950).

[4] F. E. Harris, Bull. Am. Phys. Soc. 6, 345 (1961); Rev. Mod. Phys. 35, 558 (1963).

[5] P. M. Morse and H. Feshbach, Methods of Theoretical Physics, p. 1263 (McGraw-Hill Book Co., New York, N.Y., 1953).

[6] P. M. Morse and H. Feshbach, op. cit. p. 1274.

[7] H. A. Bethe and E. E. Salpeter, Handbuch der Physik p. 266 V. 35 (Springer, Berlin, 1957); J. P. Auffray, J. K. Percus, and G. G. Ross, J. Phys. Radium (France) 23, 223 (1962).

[8] W. Magnus and F. Oberhettinger, Functions of Mathematical Physics, p. 53 (Chelsea, New York, 1954).

[9] A. R. Edmonds, Angular Momentum in Quantum Mechanics, ch 4 (Princeton, 1957).

[10] E. W. Hobson, The Theory of Spherical and Ellipsoidal Harmonics, p. 140 (Cambridge, 1931).

[11] M. E. Rose, Elementary Theory of Angular Momentum, p. 34 (John Wiley \& Sons Inc., New York, N.Y., 1957).

[12] W. Magnus and F. Oberhettinger, op. cit. p. 54.

[13] W. Magnus and F. Oberhettinger, op. cit. p. 35.

[14] Bateman Manuscript Project, Tables of Integral Transforms, vol. 2, p. 88, eq. 8.19.6 (McGraw-Hill Book Co., New York, N.Y., 1954).

[15] Bateman Manuscript Project, Higher Transcendental Functions, vol. 1, sec. 5.3 (McGraw-Hill Book Co., New York, N.Y., 1953).

[16] W. Magnus and F. Oberhettinger, op. cit. p. 84.

[17] W. Magnus and F. Oberhettinger, op. cit. p. 21.

[18] W. Magnus and F. Oberhettinger, op. cit. p. 96.

[19] S. Fraga and B. J. Ransil, J. Chem. Phys. 35, 1967 (1961) obtain E=-1.3349 a.u. for a 3 term Slater basis set while an 8 term Gaussian set yields an energy of -1.13345 a.u.

(Paper 68B1-114) 